\title{
Effectiveness of Laparoscopic Ovarian Drilling (LOD) on restoration of menstrual cycles, ovulation and pregnancy in clomiphene citrate resistant women with PCOS
}

\author{
Rama Singh Chundawat*, Arun Gupta
}

Department of Obstetrics and Gynecology, Geetanjali Medical College, Udaipur, Rajasthan, India

Received: 21 September 2017

Accepted: 27 October 2017

\section{*Correspondence:}

Dr. Rama Singh Chundawat,

E-mail: drramasingh84@gmail.com

Copyright: () the author(s), publisher and licensee Medip Academy. This is an open-access article distributed under the terms of the Creative Commons Attribution Non-Commercial License, which permits unrestricted non-commercial use, distribution, and reproduction in any medium, provided the original work is properly cited.

\section{ABSTRACT}

Background: Anovulatory infertility is present in $75 \%$ of patients with polycystic ovarian syndrome (PCOS), in addition to oligomenorrhoea and signs and symptoms of hyperandrogenism. This study was performed to evaluate the role of laparoscopic ovarian drilling (LOD) on ovulation, restoring menstrual cycles and pregnancy in clomiphene resistant women with PCOS.

Methods: This prospective cohort study included 50 women who were diagnosed with PCOS and anovulatory infertility. These patients had already tried Clomiphene citrate for conception and did not ovulate, underwent LOD between $1^{\text {st }}$ September 2009 until February 2011 in the Department of Gynecology and Obstetrics, at the Panna Dhay Mahila Chikitasalya, Udaipur. The major outcome measures include spontaneous ovulation, menstrual regularity after LOD and pregnancy rates.

Results: Among 50 patients $33(66 \%)$ started to menstruate regularly and $60 \%$ ovulated spontaneously, of them 24 (48\%) conceived.

Conclusions: Women with PCOS respond favourably to LOD.

Keywords: LOD, PCOS

\section{INTRODUCTION}

Polycystic ovarian syndrome is the most common cause of anovulatory infertility. According to the Rotterdam criteria, PCOS is characterised by a combination of oligo/amenorrhoea, clinical or endocrine signs of hyperandrogenism and polycystic ovaries. ${ }^{1}$

There are several treatment options for PCOS related anovulatory infertility. First line of treatment is usually clomiphene citrate, but when it fails to result in conception, gonadotrophins or laparoscopic ovarian drilling (LOD) may be used as a second line treatment. Gonadotrophin use requires extensive monitoring because of high sensitivity of polycystic ovaries to exogenous gonadotrophins, with the risk of multiple follicle development leading to termination of cycle, ovarian hyperstimulation syndrome or multiple pregnancies. ${ }^{2}$ Laparoscopic ovarian drilling, on the other hand, involves a single procedure that has minimal morbidity and can lead to consecutive ovulations with minimal risks of multiple pregnancies. ${ }^{3}$ Sensitivity to clomiphene citrate increases after this treatment. ${ }^{4}$ Adnexal adhesions and reduced ovarian reserve are considered two probable hazards of LOD. The clinical response to LOD seems to be thermal energy dose dependent. LOD with four punctures delivers 640 joules/ovary widely accepted by many investigators. However, the optimal amount of electrosurgical energy 
needed during LOD to achieve maximum reproductive outcome without risks is uncertain. ${ }^{5}$

The aim of this study was to evaluate the efficacy of LOD in the treatment of anovulatory infertility in PCOS.

\section{METHODS}

During the period from September 2009 through February 2011, fifty patients of PCOS who under-went LOD using diathermy were included in this study.

\section{Inclusion criteria}

Women between 20-40 years age and BMI between 2032 , treated with clomiphene citrate but failed to ovulate or get pregnant, were included in this study.

\section{Exclusion criteria}

The following patients were excluded:

Associated infertility factors such as bilateral tubal block, male factor, hyperprolactinemia, thyroid disease, diabetes.

The diagnosis of PCOS was based on the Rotterdam criteria which necessitate two of the following three features to be present for diagnosis:

- Oligomenorrhea and / or anovulation;

- Clinical and / or biochemical signs of hyperandrogenism; and

- Ultrasonic confirmation of polycystic ovaries

The hormonal profile including $\mathrm{FSH}$, LH, prolactin, thyroid levels were carried out in the MB Hospital laboratory on $3^{\text {rd }}$ day of menses or any indexed day in amenorrhoeic patients. In obese patients fasting serum insulin was also estimated. Abdominal/vaginal ultrasound was performed. Diagnosis was based on ultrasound evidence of stromal hypertrophy and more than 10 small follicles (2-9 $\mathrm{mm}$ ) arranged peripherally. Ovarian volume more than 10 was considered as part of diagnosis.

Demographic features of each patient including age, BMI, duration of infertility, type of infertility (primary or secondary), and associated clinical features of hyperandrogenism such as acne and hirsutism were noticed. Diagnostic laparoscopy was performed. Whole of the pelvis, uterus and adnexal structures were evaluated. Tubal patency was confirmed by chromopertubation test and LOD was done only when tubes were patent. The technique of LOD used in this hospital is three puncture approach Laparoscopy. Electrosurgical units set at monopolar coagulation current at 40 watts was used. A laparoscopic ovarian diathermy needle made of stainless steel and measuring $8 \mathrm{~mm}$ in length and $2 \mathrm{~mm}$ in diameter. Duration of each penetration was 5 seconds. About 3-5 punctures, $2 \mathrm{~mm}$ in diameter and 7-8 $\mathrm{mm}$ in depth, were made.

\section{Post-operative monitoring}

Following ovarian drilling women were asked to keep a record of their menstrual cycle. If she started to menstruate regularly serial ultrasound was performed to confirm the ovulation. If ovulation was not confirmed, clomiphene citrate was started as an adjuvant at 6-8 weeks after surgery. If ovulation was achieved either spontaneously or with the help of clomiphene citrate, patients were followed up until they conceived or until 12 months after LOD.

Informed consent of the patients was taken.

\section{RESULTS}

A group of 50 women with the diagnosis of PCOS underwent laparoscopic ovarian drilling during the study period. Demographic features of the 50 patients are presented in the Table 1. Mean age of the patients was $27.2 \pm 3.4$ and BMI was $27.76 \pm 3.5 \mathrm{~kg} / \mathrm{m}^{2}$ (SD 3.5) that ranged between 23.5 to $31.2 \mathrm{~kg} / \mathrm{m}^{2}$. Mean $\mathrm{LH}$ level was 11.2 IU/L (range 3.8-15.9). Forty-four (88\%) patients had ultrasound features typical of PCOS.

Table 1: The characteristics of 50 PCOS women who had LOD for anovulatory infertility.

\begin{tabular}{|llll|}
\hline Characteristic & N & Mean & Range \\
\hline Age (years) & 50 & 27.2 & $20-34$ \\
\hline BMI & 50 & 27.76 & $23.7-32$ \\
\hline Duration of Infertility (years) & 50 & 3.32 & $3-9$ \\
\hline Serum LH (IU/L) & 50 & 11.48 & $5.2-15.9$ \\
\hline Serum FSH (IU/I) & 50 & 5.2 & $1.2-8.2$ \\
\hline Menstrual cycle pattern & N & $\%$ & \\
\hline Regular & 3 & 6 & \\
\hline Oligomenorrhoea & 32 & 64 & \\
\hline Amenorrhoea & 15 & 30 & \\
\hline Hirsutism & & & \\
\hline Yes & 37 & 74 & \\
\hline No & 13 & 26 & \\
\hline Acne & & & \\
\hline Yes & 16 & 32 \\
\hline No & 33 & 66 \\
\hline Infertility & & \\
\hline Primary & 43 & 87.8 \\
\hline Secondary & 6 & 12.2 \\
\hline Ultrasound evidence of PCO & \\
\hline Atypical of PCOS & 5 & 10 \\
\hline Typical of PCOS & 45 & 90 \\
\hline FSH: Follicular stimulating hormo: & \\
\hline & & \\
\hline
\end{tabular}

Clinical features of hyperandrogenism i.e., hirsutism and acne were present in $37(74 \%)$ and 16 (32\%) respectively. Degree of hirsutism was only assessed as mild / moderate or severe. Most of these patients had either 
oligomenorrhoea $(64 \%)$ or amenorrhoea (30\%). Only $6 \%$ patients had regular menstrual cycle.

The Table 2 depicts the outcome in terms of cycle regularity, ovulation and pregnancy. Thirty-three patients (66\%) resumed regular menstruation after LOD whereas $17(34 \%)$ patients failed to do so. After LOD spontaneous ovulation occurred in $60 \%$ patients; whereas $40 \%$ did so after supplemental CC. Total pregnancies achieved after LOD were 24 (48\%), out of these 3 resulted in miscarriage. One patient opted for MTP as she was being divorced. One patient had molar pregnancy. The patients were followed up for 1 year only and pregnancies achieved in second year or after were not included, although 2 additional pregnancies did occur between 12 to 24 months.

Table 2: Outcome measures of LOD.

\begin{tabular}{|lll|}
\hline Menstrual cycle pattern & N & $\%$ \\
\hline Regular & 33 & 66 \\
\hline Irregular & 17 & 34 \\
\hline Ovulation & & \\
\hline Spontaneous & 30 & 60 \\
\hline CC & 20 & 40 \\
\hline Pregnancy & & \\
\hline Take home baby & 21 & 42 \\
\hline Miscarriage & 3 & 6 \\
\hline Ectopic & 0 & \\
\hline Unsuccessful & 26 & 52 \\
\hline CC: Clomiphene citrate & \\
\hline
\end{tabular}

\section{DISCUSSION}

The most plausible mechanisms of action of LOD are the destruction of ovarian follicles and a part of the ovarian stroma, inducing a reduction of serum androgens and inhibin levels, which results in an increase of FSH and restores the ovulation function. ${ }^{6}$ LOD may also increase ovarian blood flow, allowing a high delivery of gonadotrophins and post-surgical local growth factors. An improvement of insulin sensitivity after LOD has also been suggested. ${ }^{6,7}$ In the present study $66 \%$ of the patients achieved a regular menstrual cycle whereas for rest $34 \%$ menses remained irregular. We achieved a spontaneous ovulation rate of $60 \%$ and rest $40 \%$ ovulated after adding $\mathrm{CC}$ i.e. all of the patients who were resistant to $\mathrm{CC}$ defined as those who failed to ovulate after $150 \mathrm{mg}$ of $\mathrm{CC}$, ovulated after LOD.

Some studies have quoted higher ovulation rates i.e. around $80 \%$. Difference in response may be attributed to the use of other forms of energy modalities such as $\mathrm{CO} 2$ laser and Argon laser. Hassan E conducted a similar study of 181 CC resistant PCOS patients in which $88 \%$ obtained a regular menses, spontaneous ovulation of $70 \%$ and pregnancy rate of $83 \%$. A much higher pregnancy rate in his study may be attributed to a longer period of follow up i.e. 2 years in his study. In this study follow up was only for one year and pregnancies obtained in second year were not included, although 2 additional pregnancies did occur between 12 to 24 months. Moreover, we did not use gonadotropin for ovulation stimulation which was used by Eftekhar. The same was the reason for multiple pregnancy in his study. ${ }^{9}$

About 20-30\% of the patients do not respond to LOD. It may be due to insufficient dose of thermal energy, but studies reveal that LOD increase the amount of FSH and only minimal amount of thermal energy is required so dose of thermal energy has to be optimized in accordance with the ovarian volume. Another possible explanation is inherent resistance of ovaries to the effects of LOD. Another cause may be hyperprolactenemia observed in some patient after LOD. ${ }^{10}$

\section{CONCLUSION}

LOD is effective in restoring menstrual cycles and spontaneous ovulation in PCOS patients. It also renders ovaries sensitive to $\mathrm{CC}$ and can be used as an alternative to gonadotrophins in $\mathrm{CC}$ resistant PCOS patients with advantages of inducing monofollicular stimulation avoiding the risk of multiple pregnancy and OHSS and cost effective as intensive monitoring is not required.

\section{Funding: No funding sources}

Conflict of interest: None declared

Ethical approval: The study was approved by the Institutional Ethics Committee

\section{REFERENCES}

1. Bachelot A. Polycystic ovarian syndrome: clinical and biological diagnosis. Ann Biol Clin (Paris). 2016;74(6);661-7.

2. Jacobs HS, Agrawal R. Complications of ovarian stimulation. Baillieres Clin Obstet Gynecol. 1998;12:565-79.

3. Homburg R, Howles CM. Low dose FSH therapy for anovulatory infertility associated with polycystic ovarian syndrome: rationale, results, reflections, and refinements. Human Reprod Updates. 1999;5:493-9.

4. Donesky BW, Adashi EY. Surgically induced ovulation in the polycystic ovary syndrome: Wedge resection revisited in the age of laparoscopy. Fertile Sterile. 1995:63.

5. Badawy A, Elnashar A. Treatment options for polycystic ovary syndrome. Int J Women's Health Infertility. 2011;3:25-35.

6. Amer SA, Li TC, Ledger WI. Ovulation induction using laparoscopic ovarian drilling in women with polycystic ovarian syndrome: Predictors of success. Human Reprod. 2004;3:1-6.

7. Api M. Is ovarian reserve diminished after laparoscopic ovarian drilling?. Gynecol Endocrinol. (2009)25:159-65.

8. Farquhar C, Brown J, Marjoribanks J. Laparoscopic drilling by diathermy or laser for ovulation induction 
in anovulatory polycystic ovary syndrome. Cochrane Database Syst Rev. 2012;6.

9. Al-Ojaimi EH. Pregnancy outcomes after laparoscopic ovarian drilling in women with polycystic ovarian syndrome. Saudi Med J 2006;31:519-25.

10. Parsanezhad ME, Alborzi S, Zolghadril J, ParsaNezhad M, Keshavarzi G, Omrani GR, et al. Hyperprolactinemia after laparoscopic ovarian drilling: an unknown phenomenon. Reprod Biol Endocrinol. 2005;3(1):31.

Cite this article as: Chundawat RS, Gupta A. Effectiveness of Laparoscopic Ovarian Drilling (LOD) on restoration of menstrual cycles, ovulation and pregnancy in clomiphene citrate resistant women with PCOS. Int J Reprod Contracept Obstet Gynecol 2017;6:5425-8. 